\title{
Perturbation behavior of a multiple eigenvalue in generalized Hermitian eigenvalue problems
}

\author{
Yuji Nakatsukasa
}

Received: 18 November 2009 / Accepted: 16 January 2010 / Published online: 29 January 2010

(C) The Author(s) 2010. This article is published with open access at Springerlink.com

\begin{abstract}
We show that a multiple eigenvalue has different sensitivities under perturbations in a generalized Hermitian eigenvalue problem. Our result provides a solution to a question raised by Stewart and Sun. We also show how this difference of sensitivities plays a role in the eigenvalue forward error analysis after the Rayleigh-Ritz process, for which we present an approach that provides tight bounds.
\end{abstract}

Keywords Multiple eigenvalue · Perturbation - Generalized Hermitian eigenvalue problem

Mathematics Subject Classification (2000) $15 \mathrm{~A} 22 \cdot 15 \mathrm{~A} 42 \cdot 65 \mathrm{~F} 15$

\section{Introduction}

This paper is motivated by a question raised in [15, p. 300], where it was pointed out that multiple eigenvalues of a Hermitian positive definite matrix pencil $A-\lambda B(A, B$ are Hermitian and $B$ is positive definite) tend to behave differently under perturbations. Specifically, they consider pencils such as

$$
A-\lambda B=\left(\begin{array}{cc}
2 & 0 \\
0 & 20000
\end{array}\right)-\lambda\left(\begin{array}{cc}
1 & 0 \\
0 & 10000
\end{array}\right),
$$

which has a multiple eigenvalue $\lambda=2$ of multiplicity 2 . Interestingly, one of the two eigenvalues reacts more sensitively to perturbations than the other does. For example, define the perturbed pencil by $A+E-\lambda(B+F)$ where $E$ and $F$ are random Hermitian perturbation matrices with $\|E\|_{2},\|F\|_{2} \leq 10^{-2}$. We tested for $10^{4}$

Communicated by Axel Ruhe.

Y. Nakatsukasa $(\bowtie)$

Department of Mathematics, University of California, Davis, One Shields Avenue,

Davis, CA 95616, USA

e-mail: ynakatsukasa@ucdavis.edu 
such random matrices (details in Sect. 2.5), and observed that one eigenvalue of $A+E-\lambda(B+F)$ always lies in the interval $\left[2-1.6 \times 10^{-4}, 2+1.6 \times 10^{-4}\right]$, while the other can be more perturbed and lies in $\left[2-2.0 \times 10^{-2}, 2+2.0 \times 10^{-2}\right]$.

There seems to be a clear sensitivity difference between the two multiple eigenvalues, but a theoretical explanation for this behavior has remained an open problem. For example, a general Weyl-type perturbation result [10] applied to (1.1) only gives $|\lambda-2| \leq 3.03 \times 10^{-2}$, which is a tight bound for the more sensitive eigenvalue, but does not tell anything about the insensitive one.

The purpose of this paper is to provide a reasoning for this behavior. Theorem 2.2, our main result, gives $k$ different perturbation bounds for a multiple eigenvalue of a Hermitian positive definite pencil $A-\lambda B$ of multiplicity $k$. Applying the theorem to the pencil (1.1) lets us explain theoretically that the two eigenvalues have different perturbation behaviors.

The fact that multiple eigenvalues react differently to perturbations has several practical consequences. One example we discuss here is the forward error analysis of computed multiple eigenvalues (Ritz values) obtained by the Rayleigh-Ritz process. We show how the different sensitivities of a multiple eigenvalue plays a role here, and present an approach that yields $k$ different error bounds for a multiple Ritz value of multiplicity $k$.

The structure of this paper is as follows. In Sect. 2 we present our main result, which gives $k$ different perturbation bounds for a multiple eigenvalue of a Hermitian positive definite pencil. In Sect. 3 we describe an approach that provides refined forward error bounds for a computed multiple eigenvalue after the Rayleigh-Ritz process.

Notations: We denote the $i$ th smallest eigenvalue of a Hermitian positive definite pencil (or a Hermitian matrix) by $\lambda_{i}$, and $\sigma_{i}(X)$ denotes the $i$ th smallest singular value of a matrix $X . I_{n}$ denotes an $n$-by- $n$ identity matrix. We only deal with the Hermitian positive definite case, so whenever we write $A-\lambda B, A$ and $B$ are Hermitian and $B$ is positive definite. $\|\cdot\|_{2}$ is the matrix spectral norm and $\|v\|_{B}=\sqrt{v^{H} B v}$ for a vector $v$ and a positive definite matrix $B$.

\section{Perturbation bounds for multiple eigenvalues in $A x=\lambda B x$}

Suppose a Hermitian positive definite pencil $A-\lambda B$ has a multiple eigenvalue $\lambda_{0}$ of multiplicity $k$. In this section we consider a perturbed Hermitian positive definite pencil $(A+E)-\lambda(B+F)$. We are interested in the eigenvalues of $(A+E)-\lambda(B+F)$ that are close to $\lambda_{0}$ : how the multiple eigenvalue $\lambda_{0}$ is perturbed. Our goal is to give $k$ different perturbation bounds, that is, to derive $0 \leq b_{1} \leq b_{2} \leq \cdots \leq b_{k}$ such that there are at least $i$ eigenvalues of $(A+E)-\lambda(B+F)$ that lie in $\left[\lambda_{0}-b_{i}, \lambda_{0}+b_{i}\right]$.

\subsection{Preliminary results}

We will use Theorem 2.1 below, whose proof needs the following Lemma. Both results are based on [12]. 
Lemma 2.1 Suppose $A-\lambda(B+\Delta B)$ and $A-\mu_{i} \Delta B-\lambda B$ are Hermitian positive definite pencils. If $\mu_{i}$ is the ith eigenvalue of the first pencil, then it is also the ith eigenvalue of the second pencil.

Proof Since the two pencils are Hermitian positive definite, there exist nonsingular $Z_{1}$ and $Z_{2}$ such that $Z_{1}^{H} A Z_{1}=\Lambda_{1}, Z_{1}^{H}(B+\Delta B) Z_{1}=I, Z_{2}^{H}\left(A-\mu_{i} \Delta B\right) Z_{2}=\Lambda_{2}$ and $Z_{2}^{H} B Z_{2}=I$, where $\Lambda_{1}$ and $\Lambda_{2}$ are diagonal matrices containing the eigenvalues [4, Sect. 8.7].

Then, denoting $M=A-\mu_{i}(B+\Delta B)$, we have $Z_{1}^{H} M Z_{1}=\Lambda_{1}-\mu_{i} I$ and $Z_{2}^{H} M Z_{2}=\Lambda_{2}-\mu_{i} I$. Note that by assumption both matrices have a zero eigenvalue, which is the $i$ th eigenvalue of the first matrix. Since by Sylvester's law of inertia [3, Sect. 5.2], $Z_{1}^{H} M Z_{1}$ and $Z_{2}^{H} M Z_{2}$ have the same inertia, it follows that 0 is the $i$ th eigenvalue of both matrices, so $\mu_{i}$ is the $i$ th eigenvalue of both $\Lambda_{1}$ and $\Lambda_{2}$.

Theorem 2.1 Suppose $A-\lambda B$ and $(A+\Delta A)-\lambda(B+\Delta B)$ are Hermitian positive definite pencils, and let $\lambda_{1} \leq \lambda_{1} \leq \cdots \leq \lambda_{n}$ be the eigenvalues of $A-\lambda B$ and let $\widehat{\lambda}_{1} \leq \widehat{\lambda}_{1} \leq \cdots \leq \widehat{\lambda}_{n}$ be the eigenvalues of $(A+\Delta A)-\lambda(B+\Delta B)$. Then,

$$
\left|\widehat{\lambda}_{i}-\lambda_{i}\right| \leq\left\|(B+\Delta B)^{-1}\right\|_{2}\left\|\Delta A-\lambda_{i} \Delta B\right\|_{2}
$$

and

$$
\left|\widehat{\lambda}_{i}-\lambda_{i}\right| \leq\left\|B^{-1}\right\|_{2}\left\|\Delta A-\widehat{\lambda}_{i} \Delta B\right\|_{2}
$$

for all $1 \leq i \leq n$.

Proof We prove (2.2). Suppose

$$
(A+\Delta A) \widehat{x_{i}}=\widehat{\lambda_{i}}(B+\Delta B) \widehat{x_{i}} .
$$

This is equivalent to

$$
\left(A+\Delta A-\widehat{\lambda}_{i} \Delta B\right) \widehat{x}_{i}=\widehat{\lambda}_{i} B \widehat{x}_{i} .
$$

Equation (2.4) means the pencil $\left(A+\Delta A-\widehat{\lambda}_{i} \Delta B\right)-\lambda B$ has an eigenpair $\left(\widehat{\lambda}_{i}, \widehat{x}_{i}\right)$. Moreover, using Lemma 2.1 by substituting $A+\Delta A$ into $A$ and $\widehat{\lambda_{i}}$ into $\mu_{i}$, we know that $\widehat{\lambda}_{i}$ is the $i$ th eigenvalue. Equation (2.4) can be transformed into a standard Hermitian eigenvalue problem by premultiplying $B^{-1 / 2}$ (the matrix square root of $B^{-1}$ [5, Chap. 5]):

$$
B^{-1 / 2}\left(A+\Delta A-\widehat{\lambda}_{i} \Delta B\right) B^{-1 / 2} y_{i}=\widehat{\lambda}_{i} y_{i},
$$

where we defined $y_{i}=B^{1 / 2} \widehat{x_{i}}$. Noting that the matrix $B^{-1 / 2} A B^{-1 / 2}$ and the pencil $A-\lambda B$ have the same eigenvalues, we conclude by using Weyl's theorem that

$$
\left|\lambda_{i}-\widehat{\lambda}_{i}\right| \leq\left\|B^{-1 / 2}\left(\Delta A-\widehat{\lambda}_{i} \Delta B\right) B^{-1 / 2}\right\|_{2} \leq\left\|B^{-1}\right\|_{2}\left\|\Delta A-\widehat{\lambda}_{i} \Delta B\right\|_{2},
$$

which is (2.2). Equation (2.1) can be obtained similarly by starting with $A x_{i}=\lambda_{i} B x_{i}$ in (2.3).

Note that if $\|\Delta B\|_{2}<\lambda_{\min }(B)$ then $(A+\Delta A)-\lambda(B+\Delta B)$ is a Hermitian positive definite pencil. 


\subsection{Choosing eigenvectors}

Since $A-\lambda B$ is a Hermitian positive definite pencil with a multiple eigenvalue $\lambda_{0}$ of multiplicity $k$, there exists a nonsingular matrix $X$ such that

$$
X^{H} A X=\Lambda=\operatorname{diag}\left(\lambda_{0}, \ldots, \lambda_{0}, \lambda_{k+1}, \ldots, \lambda_{n}\right), \quad X^{H} B X=I_{n},
$$

where $\lambda_{k+i} \neq \lambda_{0}$ for $i \geq 1$. The columns of $X$ are the right eigenvectors of $A-\lambda B$, and the first $k$ columns are the eigenvectors corresponding to the multiple eigenvalue $\lambda_{0}$. It is important to note that $X$ is not unique, in that there is freedom of unitary transformations in the first $k$ columns of $X$. Specifically, for any unitary matrix $Q \in \mathbb{C}^{k \times k}, X$ can be replaced by $X \cdot \operatorname{diag}\left(Q, I_{n-k}\right)$, and (2.5) still holds. Among the possible choices of $X$, considering the following specific choice $X_{0}$ will be essential for our analysis below.

Choice of $X_{0}$ : Among the possible $X$ that satisfy (2.5), we choose $X_{0}$ such that the first $k$ columns of $X_{0}=\left[x_{1}, x_{2}, \ldots, x_{k}, \ldots, x_{n}\right]$ are chosen so that they are mutually orthogonal, that is,

$$
\left[x_{1}, x_{2}, \ldots, x_{k}\right]=U \Sigma I_{k}
$$

is the SVD, so that $U=\left[u_{1}, u_{2}, \ldots, u_{k}\right] \in \mathbb{C}^{n \times k}, U^{H} U=I_{k}$ and $\Sigma=\operatorname{diag}\left(\sigma_{1}\right.$, $\ldots, \sigma_{k}$ ) with $0<\sigma_{1} \leq \cdots \leq \sigma_{k}$.

Note that $\Sigma$ is unique for any choice of unitary matrix $Q$ as shown above. As for obtaining such $X_{0}$, given an arbitrary $\hat{X}$ that satisfies $(2.5)$, we can get $X_{0}$ by first computing the SVD of the first $k$ columns of $\hat{X}: \hat{X}(:, 1: k)=U \Sigma V^{H}$, and then letting $X_{0}(:, 1: k)=\hat{X}(:, 1: k) V=U \Sigma$ and $X_{0}(:, k+1: n)=\hat{X}(:, k+1: n)$ (we use MATLAB notation, denoting by $\hat{X}(:, 1: k)$ the first $k$ columns of $\hat{X})$.

Now, given an integer $t(\leq k)$, write

$$
X_{0}^{H} E X_{0}=\left[\begin{array}{cc}
E_{11}^{(t)} & E_{12}^{(t)} \\
E_{21}^{(t)} & O
\end{array}\right]+\left[\begin{array}{cc}
O & O \\
O & E_{22}^{(t)}
\end{array}\right] \equiv E_{1}^{(t)}+E_{2}^{(t)},
$$

and

$$
X_{0}^{H} F X_{0}=\left[\begin{array}{cc}
F_{11}^{(t)} & F_{12}^{(t)} \\
F_{21}^{(t)} & O
\end{array}\right]+\left[\begin{array}{cc}
O & O \\
O & F_{22}^{(t)}
\end{array}\right] \equiv F_{1}^{(t)}+F_{2}^{(t)},
$$

where $E_{11}^{(t)}$ and $F_{11}^{(t)}$ are $t$-by- $t$. Note that the two pencils $(A+E)-\lambda(B+F)$ and $\left(\Lambda+E_{1}^{(t)}+E_{2}^{(t)}\right)-\lambda\left(I+F_{1}^{(t)}+F_{2}^{(t)}\right)$ are congruent, so they have the same eigenvalues.

Our next task is to bound $\left\|E_{i}^{(t)}\right\|_{2}$ and $\left\|F_{i}^{(t)}\right\|_{2}(i=1,2)$. We shall show that $\left\|E_{1}^{(t)}\right\|_{2}$ and $\left\|F_{1}^{(t)}\right\|_{2}$ are small for $t$ such that $\sigma_{t}$ is small. This in turn implies that a small interval exists that traps $t$ eigenvalues of $(A+E)-\lambda(B+F)$. 
2.3 Bounding $\left\|E_{1}^{(t)}\right\|_{2}$ and $\left\|F_{1}^{(t)}\right\|_{2}$

To bound $\left\|E_{1}^{(t)}\right\|_{2}$, we use

$$
\left\|E_{1}^{(t)}\right\|_{2}=\left\|\left[\begin{array}{cc}
E_{11}^{(t)} & E_{12}^{(t)} \\
E_{21}^{(t)} & O
\end{array}\right]\right\|_{2} \leq\left\|\left[\begin{array}{cc}
0 & E_{12}^{(t)} \\
E_{21}^{(t)} & 0
\end{array}\right]\right\|_{2}+\left\|\left[\begin{array}{cc}
E_{11}^{(t)} & 0 \\
0 & 0
\end{array}\right]\right\|_{2}
$$

The first term can be bounded by

$$
\left\|\left[\begin{array}{cc}
0 & E_{12}^{(t)} \\
E_{21}^{(t)} & 0
\end{array}\right]\right\|_{2}=\left\|E_{12}^{(t)}\right\|_{2} \leq \sigma_{t} \sqrt{\left\|B^{-1}\right\|_{2}}\|E\|_{2},
$$

because $E_{12}^{(t)}=\Sigma_{t} U_{t}^{H} E X_{0}(:, t+1: n)$, where $U_{t}=\left[u_{1}, \ldots, u_{t}\right], \Sigma_{t}=\operatorname{diag}\left(\sigma_{1}\right.$, $\ldots, \sigma_{t}$ ), so using $\left\|\Sigma_{t}\right\|_{2}=\sigma_{t}$ and $\left\|X_{0}\right\|_{2}=\sqrt{\left\|B^{-1}\right\|_{2}}$ (which follows from $B=$ $X_{0}^{-H} X_{0}^{-1}$ ), we get $\left\|E_{12}^{(t)}\right\|_{2} \leq\left\|\Sigma_{t}\right\|_{2}\left\|U_{t}\right\|_{2}\|E\|_{2}\left\|X_{0}\right\|_{2}=\sigma_{t} \sqrt{\left\|B^{-1}\right\|_{2}}\|E\|_{2}$.

The second term of (2.9) can be bounded by

$$
\left\|\left[\begin{array}{cc}
E_{11}^{(t)} & 0 \\
0 & 0
\end{array}\right]\right\|_{2}=\left\|E_{11}^{(t)}\right\|_{2} \leq \sigma_{t}^{2}\|E\|_{2}
$$

because $E_{11}^{(t)}=\Sigma_{t} U_{t}^{H} E U_{t} \Sigma_{t}$, from which we get $\left\|E_{11}^{(t)}\right\|_{2} \leq\left\|\Sigma_{t}\right\|_{2}^{2} \cdot\left\|U_{t}\right\|_{2}^{2} \cdot\|E\|_{2}=$ $\sigma_{t}^{2}\|E\|_{2}$.

Substituting these into (2.9) yields

$$
\left\|E_{1}^{(t)}\right\|=\left\|\left[\begin{array}{cc}
E_{11}^{(t)} & E_{12}^{(t)} \\
E_{21}^{(t)} & O
\end{array}\right]\right\|_{2} \leq \sigma_{t}\left(\sqrt{\left\|B^{-1}\right\|_{2}}+\sigma_{t}\right)\|E\|_{2}
$$

Similarly, we can bound $\left\|F_{1}^{(t)}\right\|_{2}$ by

$$
\left\|F_{1}^{(t)}\right\|_{2}=\left\|\left[\begin{array}{cc}
F_{11}^{(t)} & F_{12}^{(t)} \\
F_{21}^{(t)} & O
\end{array}\right]\right\|_{2} \leq \sigma_{t}\left(\sqrt{\left\|B^{-1}\right\|_{2}}+\sigma_{t}\right)\|F\|_{2} .
$$

As for bounding $\left\|E_{2}^{(t)}\right\|_{2}$ and $\left\|F_{2}^{(t)}\right\|_{2}$, it is straightforward to see from (2.7) and (2.8) that $\left\|E_{2}^{(t)}\right\|_{2}$ and $\left\|F_{2}^{(t)}\right\|_{2}$ satisfy

$$
\begin{aligned}
& \left\|E_{2}^{(t)}\right\|_{2} \leq\left\|X_{0}^{H} E X_{0}\right\|_{2} \leq\left\|B^{-1}\right\|_{2}\|E\|_{2}, \\
& \left\|F_{2}^{(t)}\right\|_{2} \leq\left\|X_{0}^{H} F X_{0}\right\|_{2} \leq\left\|B^{-1}\right\|_{2}\|F\|_{2} .
\end{aligned}
$$




\subsection{Main result}

Now we are ready to state our main result.

Theorem 2.2 Suppose that $A-\lambda B$ and $(A+E)-\lambda(B+F)$ are $n$-by-n Hermitian positive definite pencils, and that $A-\lambda B$ has a multiple eigenvalue $\lambda_{0}$ of multiplicity $k$. Let $X$ be a nonsingular matrix that satisfies (2.5). Denote by $0<\sigma_{1} \leq \sigma_{2} \leq \cdots \leq \sigma_{k}$ the singular values of $X(:, 1: k)$, the first $k$ columns of $X$. Then, for any integer $t$ such that $1 \leq t \leq k,(A+E)-\lambda(B+F)$ has at least $t$ eigenvalues $\widehat{\hat{\lambda}_{i}}(i=1,2, \ldots, t)$ that satisfy

$$
\left|\widehat{\lambda_{i}}-\lambda_{0}\right| \leq \sigma_{t}\left(\sqrt{\left\|B^{-1}\right\|_{2}}+\sigma_{t}\right) \frac{\|E\|_{2}+\left|\lambda_{0}\right|\|F\|_{2}}{1-\left\|B^{-1}\right\|_{2}\|F\|_{2}} .
$$

Proof Recall that the pencils $(A+E)-\lambda(B+F)$ and $\left(\Lambda+E_{1}^{(t)}+E_{2}^{(t)}\right)-\lambda(I+$ $\left.F_{1}^{(t)}+F_{2}^{(t)}\right)$ have the same eigenvalues, and note that the pencil $\left(\Lambda+E_{2}^{(t)}\right)-\lambda(I+$ $F_{2}^{(t)}$ ) has a multiple eigenvalue $\lambda_{0}$ of multiplicity (at least) $t$. We apply Theorem 2.1 by regarding $\left(\Lambda+E_{1}^{(t)}+E_{2}^{(t)}\right)-\lambda\left(I+F_{1}^{(t)}+F_{2}^{(t)}\right)$ as a perturbed pencil of $(\Lambda+$ $\left.E_{2}^{(t)}\right)-\lambda\left(I+F_{2}^{(t)}\right)$. Then we see that the pencil $\left(\Lambda+E_{1}^{(t)}+E_{2}^{(t)}\right)-\lambda\left(I+F_{1}^{(t)}+F_{2}^{(t)}\right)$ has at least $t$ eigenvalues $\widehat{\lambda_{i}}(i=1,2, \ldots, t)$ that satisfy

$$
\begin{aligned}
\left|\widehat{\lambda_{i}}-\lambda_{0}\right| & \leq \frac{\left\|E_{1}^{(t)}\right\|_{2}+\left|\lambda_{0}\right|\left\|F_{1}^{(t)}\right\|_{2}}{\lambda_{\min }\left(I+F_{1}^{(t)}+F_{2}^{(t)}\right)} \\
& \leq \frac{\sigma_{t}\left(\sqrt{\left\|B^{-1}\right\|_{2}}+\sigma_{t}\right)\|E\|_{2}+\left|\lambda_{0}\right| \sigma_{t}\left(\sqrt{\left\|B^{-1}\right\|_{2}}+\sigma_{t}\right)\|F\|_{2}}{1-\left\|B^{-1}\right\|_{2}\|F\|_{2}} \\
& \leq \sigma_{t}\left(\sqrt{\left\|B^{-1}\right\|_{2}}+\sigma_{t}\right) \frac{\|E\|_{2}+\left|\lambda_{0}\right|\|F\|_{2}}{1-\left\|B^{-1}\right\|_{2}\|F\|_{2}},
\end{aligned}
$$

where we used (2.10), (2.11) and $\lambda_{\min }\left(I+F_{1}^{(t)}+F_{2}^{(t)}\right) \geq 1-\left\|F_{1}^{(t)}+F_{2}^{(t)}\right\|_{2}=1-$ $\left\|X_{0}^{H} F X_{0}\right\|_{2} \geq 1-\left\|B^{-1}\right\|_{2}\|F\|_{2}$, which follows from Weyl's theorem.

We emphasize that inequality (2.13) holds for $t$ (not $k$ ) eigenvalues of $\left(\Lambda+E_{1}^{(t)}+\right.$ $\left.E_{2}^{(t)}\right)-\lambda\left(I+F_{1}^{(t)}+F_{2}^{(t)}\right)$. The upper bound in (2.13) for $t=t_{0}$ is much smaller than that for $t=k$ if $\sigma_{t_{0}} \ll \sigma_{k}$. In such a case, among the $k$ eigenvalues of $A-\lambda B$ equal to $\lambda_{0}$, there are $t_{0}$ eigenvalues that are much less sensitive than the most sensitive one.

\subsection{Simple example}

Let us return to the simple example shown in the introduction and examine the sharpness of our results. For the pencil (1.1), we formed perturbed Hermitian positive definite pencils $A+E-\lambda(B+F)$ using MATLAB version 7.4 by defining $E$ and $F$ by $\alpha\left(C^{H}+C\right)$, where the entries of the 2-by-2 matrix $C$ are random numbers in $[-1 / 2,1 / 2]$ generated by the MATLAB function rand -0.5 and $\alpha$ 
is defined by $10^{-2} \times$ rand $/\left\|C^{H}+C\right\|_{2}$ to force $\|E\|_{2},\|F\|_{2} \leq 10^{-2}$. Experimenting with $10^{4}$ such pencils, we observed that one eigenvalue is always trapped in $\left[2-1.6 \times 10^{-4}, 2+1.6 \times 10^{-4}\right]$, but the interval that traps both eigenvalues needs to be as large as $\left[2-2.0 \times 10^{-2}, 2+2.0 \times 10^{-2}\right]$.

We give an explanation for this phenomenon by using Theorem 2.2. Here $\left|\lambda_{0}\right|=2$, $\left\|B^{-1}\right\|_{2}=1,\|E\|_{2} \leq 10^{-2},\|F\|_{2} \leq 10^{-2}, \sigma_{1}=10^{-2}$ and $\sigma_{2}=1$, so letting $t=1$ in (2.13) we get

$$
\begin{aligned}
\left|\lambda_{1}-2\right| & \leq 10^{-2}\left(1+10^{-2}\right) \frac{10^{-2}+2 \times 10^{-2}}{1-1 \times 10^{-2}} \\
& =3.0606 \times 10^{-4},
\end{aligned}
$$

which means at least one eigenvalue of $A+E-\lambda(B+F)$ exists in $[2-3.1 \times$ $\left.10^{-4}, 2+3.1 \times 10^{-4}\right]$.

To bound both eigenvalues we let $t=2$, which yields

$$
\begin{aligned}
\left|\lambda_{1,2}-2\right| & \leq 1(1+1) \frac{10^{-2}+2 \times 10^{-2}}{1-1 \times 10^{-2}} \\
& =6.06 \times 10^{-2},
\end{aligned}
$$

a bound that is larger than (2.14) by more than a factor of 100 .

We observe that these bounds reflect our experiments pretty accurately. Thus we claim Theorem 2.2 is one explanation for the different behaviors of multiple eigenvalues in generalized Hermitian eigenvalue problems.

\subsection{Comparison with known results}

Here we compare Theorem 2.2 with known perturbation results for a multiple eigenvalue in generalized Hermitian eigenvalue problems. To our knowledge, no result has been known that gives different a priori perturbation bounds using only the norms of the perturbation matrices (in the case of the standard eigenvalue problem, different condition numbers of a multiple eigenvalue are derived in $[16,17])$. Here we give a comparison with known first-order perturbation approximations in [2] and [7], and see that both of them require more information than just the norms of $E$ and $F$.

Below is a special case of Corollary 2.3 in [2], when $A-\lambda B$ is a positive definite pencil that has a multiple eigenvalue.

Theorem 2.3 Let $A-\lambda B$ be a Hermitian positive definite pencil that has a multiple eigenvalue $\lambda_{0}$ of multiplicity $k$. The perturbed pencil $(A+E)-\lambda(B+F)$ has $k$ eigenvalues $\widehat{\lambda}_{i}(1 \leq i \leq k)$ such that

$$
\exp \left(-\kappa_{i}\right) \leq \frac{\widehat{\lambda}_{i}}{\lambda_{0}} \leq \exp \left(\kappa_{i}\right)
$$

where

$$
\kappa_{i}=\max _{\zeta \in[0,1]}\left|\frac{x_{i}^{H}(\zeta) E x_{i}(\zeta)}{x_{i}^{H}(\zeta) A(\zeta) x_{i}(\zeta)}-\frac{x_{i}^{H}(\zeta) F x_{i}(\zeta)}{x_{i}^{H}(\zeta) B(\zeta) x_{i}(\zeta)}\right|\left(\equiv \max _{\zeta \in[0,1]} g_{i}(\zeta)\right),
$$


where the maximum in (2.16) is taken over $\zeta$ such that $\lambda_{i}(\zeta)$ is not a multiple eigenvalue. Here we denoted $A(\zeta)=A+\zeta E, B(\zeta)=B+\zeta F$, and let $\left(\lambda_{i}(\zeta), x_{i}(\zeta)\right)$ for $1 \leq i \leq k$ be the $k$ eigenpairs such that $\lambda_{i}(0)=\lambda_{0}, \lambda_{i}(1)=\widehat{\lambda}_{i}$ and

$$
A(\zeta) x_{i}(\zeta)=\lambda_{i}(\zeta) B(\zeta) x_{i}(\zeta), \quad \zeta \in[0,1]
$$

In practice, bounds (2.15) are not available because $\kappa_{i}$ cannot be computed. [2] suggests obtaining estimates of them by approximating $\kappa_{i}$ by taking $g_{i}(\zeta)$ at a specific $\zeta$, such as $\zeta=0$, which results in first-order approximations of (2.15). Unfortunately we cannot take $\zeta=0$ here because $\lambda_{i}(0)$ is a multiple eigenvalue. Instead, we can for example compute $\widehat{\kappa}_{i}=g_{i}(1)$ for $1 \leq i \leq k$. Substituting such computed $\widehat{\kappa}_{i}$ into $\kappa_{i}$ in (2.15) yields estimates (but not bounds) of the perturbed eigenvalues $\widehat{\lambda}_{i}$, which are accurate when $E$ and $F$ are small.

To see how accurate these estimates are, let us consider again the simple example (1.1). If $E=10^{-2}\left[\begin{array}{ll}1 & 0 \\ 0 & 1\end{array}\right]$ and $F=10^{-2}\left[\begin{array}{ll}0 & 1 \\ 1 & 0\end{array}\right]$, using Theorem 2.3 by estimating each $\kappa_{i}$ by $g_{i}(1)$, we get estimates for the eigenvalues of $(A+E)-\lambda(B+F)$ : $\left|\widehat{\lambda}_{1}-2\right| \lesssim 2.998784 \times 10^{-6}$ and $\left|\widehat{\lambda}_{2}-2\right| \lesssim 9.980 \times 10^{-3}$. The true eigenvalues are $\simeq\left(2-2.998789 \times 10^{-6}, 2+1.00040 \times 10^{-2}\right)$. The estimates are much sharper than the bounds given by Theorem 2.2 (see previous section). However the estimates are not strict bounds, as seen by the fact that the estimated interval for $\widehat{\lambda}_{2}$ does not trap the true eigenvalue. More importantly, Theorem 2.3 requires the eigenvectors of a perturbed pencil, which is not available if the perturbations are unknown.

Another way to obtain approximations to the perturbed eigenvalues is to use the first-order eigenvalue perturbation expansion result, for example Theorem 4.1 in [7]. This involves computing eigenvalues of the matrix $X_{k}^{H}\left(E-\lambda_{0} F\right) X_{k}$, where $X_{k}$ is the first $k$ columns of $X$ in (2.5). Note that this also requires more information than just $\|E\|_{2}$ and $\|F\|_{2}$.

In summary, the above known results that give approximations to the multiple eigenvalue perturbations are generally sharper than the bound obtained by Theorem 2.2, but require more information of the perturbation matrices $E$ and $F$. Theorem 2.2 has the advantage that it gives a priori bounds for arbitrary $E$ and $F$ using only their norms, revealing the fact that a multiple eigenvalue has different sensitivities.

\section{Rayleigh-Ritz process}

In this section we consider the eigenvalue forward error analysis after the RayleighRitz process. In particular, our focus is on how our observation in Sect. 2 plays a role in this context.

\subsection{Preliminaries}

The Rayleigh-Ritz process is frequently used in an algorithm that computes a subset of eigenvalues and eigenvectors of a large matrix/pencil. These algorithms include Lanczos, steepest descent, conjugate gradient, LOBPCG, generalized Davidson and 
Jacobi-Davidson methods $[1,6,11,13]$. For a $N$-by- $N$ generalized Hermitian eigenvalue problem $A x=\lambda B x$, given $Y \in \mathbb{C}^{N \times m}(N \gg m)$ whose columns ideally contain the desired eigenspace, the Rayleigh-Ritz process computes approximate eigenvalues/eigenvectors by solving an $m \times m$ eigenvalue problem $Y^{H} A Y z_{i}=\theta_{i} Y^{H} B Y z_{i}$ $(i=1, \ldots, m)$, from which the approximate eigenvalues $\theta_{i}$ (Ritz values) and approximate eigenvectors $w_{i}=Y z_{i}$ (Ritz vectors) are obtained such that denoting $W=\left[\begin{array}{llll}w_{1} & w_{2} & \cdots & w_{m}\end{array}\right], W^{H} A W=\Lambda=\operatorname{diag}\left(\theta_{1}, \theta_{2}, \ldots, \theta_{m}\right)$ and $W^{H} B W=I_{m}$. We are particularly interested in the case where a multiple Ritz value (of multiplicity $k$ ) exists, i.e., when $\theta_{0} \equiv \theta_{1}=\theta_{2}=\cdots=\theta_{k}$. We also have the residual vectors

$$
r_{i}=A w_{i}-\theta_{i} B w_{i}, \quad i=1,2, \ldots, m,
$$

which are nonzero but expected to be small. Conventionally, an eigenvalue forward error analysis that bounds the closeness of the Ritz values to a true eigenvalue is obtained as follows [1]. First note that (3.1) is equivalent to

$$
B^{-1 / 2} r_{i}=\left(B^{1 / 2} A B^{-1 / 2}\right)\left(B^{1 / 2} w_{i}\right)-\theta_{i}\left(B^{1 / 2} w_{i}\right)
$$

Suppose the Ritz vectors $w_{i}(1 \leq i \leq m)$ are $B$-orthonormalized, so $\left\|w_{i}\right\|_{B}=1$. Then, it is known [11, p. 73] that there exists an eigenvalue $\lambda$ of the pencil $A-\lambda B$ such that

$$
\left|\lambda-\theta_{i}\right| \leq \frac{\left\|B^{-1 / 2} r_{i}\right\|_{2}}{\left\|B^{1 / 2} w_{i}\right\|_{2}}=\frac{\left\|r_{i}\right\|_{B^{-1}}}{\left\|w_{i}\right\|_{B}} \leq \sqrt{\left\|B^{-1}\right\|_{2}}\left\|r_{i}\right\|_{2} .
$$

A quadratic error bound

$$
\left|\lambda-\theta_{i}\right| \leq \frac{1}{g a p}\left(\frac{\left\|B^{-1 / 2} r_{i}\right\|_{2}}{\left\|B^{1 / 2} w_{i}\right\|_{2}}\right)^{2} \leq \frac{\left\|B^{-1}\right\|_{2}\left\|r_{i}\right\|_{2}^{2}}{\text { gap }}
$$

can also be used when an estimate of gap (the smallest gap between $\theta_{i}$ and any eigenvalue of $A-\lambda B$ but the one closest to $\theta_{i}$ ) is available [1, Sect. 5.7.1].

\subsection{Questions}

Bounds (3.2) and (3.3) have the following caveats, regarding the multiple Ritz value $\theta_{0}$.

1. They do not reflect the different perturbation behaviors of a multiple eigenvalue that we observed in Sect. 2.

2. They only give an interval in which at least one true eigenvalue exists, so there is no known $b_{i} \geq 0$ such that $\left[\theta_{0}-b_{i}, \theta_{0}+b_{i}\right]$ contains at least $i$ true eigenvalues for $2 \leq i \leq k$.

We use a simple example to illustrate these two issues. Consider a 4-by-4 Hermitian positive definite pencil $A-\lambda B$, defined by

$$
A=\operatorname{diag}\left(10^{4}, 1,2,2\right)+\left[\begin{array}{cc}
0 & C_{1}^{H} \\
C_{1} & 0
\end{array}\right], \quad B=\operatorname{diag}\left(10^{4}, 1,1,1\right)+\left[\begin{array}{cc}
0 & C_{2}^{H} \\
C_{2} & 0
\end{array}\right],
$$


where $C_{1}, C_{2} \in \mathbb{C}^{2 \times 2}$. When $C_{1}$ and $C_{2}$ are small, the pencil $A-\lambda B$ has two eigenvalues close to 1 and another two close to 2 . Furthermore, using Theorem 2.2 we see that among the two eigenvalues close to 1 , one has to satisfy $|\lambda-1| \lesssim$ $10^{-2}\left(\left\|C_{1}\right\|_{2}+\left\|C_{2}\right\|_{2}\right)$, while the other has the bound $|\lambda-1| \lesssim\left\|C_{1}\right\|_{2}+\left\|C_{2}\right\|_{2}$, suggesting different sensitivities.

Consider for example the case $C_{1}=0.1\left[\begin{array}{ll}1 & 1 \\ 1 & 1\end{array}\right]$ and $C_{2}=0.1\left[\begin{array}{ll}1 & 0 \\ 0 & 1\end{array}\right]$ (any choice of sufficiently small random matrices $C_{1}, C_{2}$ yields similar results for the following arguments). Suppose we have an approximate eigenspace spanned by $\left(\begin{array}{llll}1 & 0 & 0 & 0\end{array}\right)^{T}$ and $\left(\begin{array}{llll}0 & 1 & 0 & 0\end{array}\right)^{T}$. The Rayleigh-Ritz process yields the 2 -by-2 pencil $\tilde{A}-\lambda \tilde{B}$ where $\tilde{A}=$ $\tilde{B}=\operatorname{diag}\left(10^{4} 1\right)$, so the Ritz values are both 1 . The resulting $B$-orthonormalized Ritz vectors may be for example $w_{1}=\left(\begin{array}{lllll}10^{-2} & 0 & 0 & 0\end{array}\right)^{T}$ and $w_{2}=\left(\begin{array}{llll}0 & 1 & 0 & 0\end{array}\right)^{T}$, which yield the residual vectors

$$
\begin{aligned}
& r_{1}=A w_{1}-B w_{1}=\left(\begin{array}{llll}
0 & 0 & 0 & 10^{-3}
\end{array}\right)^{T}, \\
& r_{2}=A w_{2}-B w_{2}=\left(\begin{array}{llll}
0 & 0 & 10^{-1} & 0
\end{array}\right)^{T} .
\end{aligned}
$$

Hence the forward eigenvalue error bound (3.2) yields

$$
|\lambda-1| \leq \sqrt{\left\|B^{-1}\right\|_{2}}\left\|r_{1}\right\|_{2} \leq \sqrt{1 / 0.9} / 1000<1.06 \times 10^{-3},
$$

which at least one eigenvalue has to satisfy (here we used $\left\|B^{-1}\right\|_{2} \leq 1 / 0.9$, which is easily obtained by Weyl's theorem. In practical problems, estimating $\left\|B^{-1}\right\|_{2}$ is a nontrivial task, which is beyond the scope of this paper).

Note that any set of vectors written as $\left[\begin{array}{ll}w_{1} & w_{2}\end{array}\right] Q$, where $Q$ is any unitary matrix, is a pair of Ritz vectors that yields the same Ritz values. Hence one may instead have as Ritz vectors for example $\widetilde{w}_{1}=\left(\frac{1}{100 \sqrt{2}} \frac{1}{\sqrt{2}} 00\right)^{T}$ and $\widetilde{w}_{2}=\left(\frac{1}{100 \sqrt{2}}-\frac{1}{\sqrt{2}} 00\right)^{T}$. In this case, the residual vectors become

$$
\widetilde{r}_{1}=\frac{1}{\sqrt{2}}\left(\begin{array}{llll}
0 & 0 & 10^{-1} & 10^{-3}
\end{array}\right), \quad \widetilde{r}_{2}=\frac{1}{\sqrt{2}}\left(\begin{array}{llll}
0 & 0 & -10^{-1} & 10^{-3}
\end{array}\right)^{T} .
$$

Applying these to (3.2) yields only $|\lambda-1| \leq 7.5 \times 10^{-2}$, which is a much looser bound than (3.6). Now the obvious question is, how can we ensure to choose the "right" Ritz vectors so we have a "good" bound such as (3.6)? This is the first question we raised.

The second question concerns the eigenvalue that is more sensitive to perturbations. It is important to note that the union of 2 bounds using $r_{1}$ and $r_{2}$ in (3.5) or (3.7) does not necessarily bound two eigenvalues, as is warned in [11, Sect. 11.5]. How can we obtain a bound that is guaranteed to contain two eigenvalues? And for a general pencil, if a multiple Ritz value has multiplicity $k$, can we get $k$ different bounds to reflect the different sensitivities?

\subsection{Choosing the "right" Ritz vectors}

This subsection answers the above two questions. We consider the issue of choosing the set of "right" Ritz vectors $W=\left\{w_{1}, w_{2}, \ldots, w_{k}\right\}$ for a multiple Ritz value $\theta_{0}$ of 
multiplicity $k$. Here, 'right' means the particular choice of Ritz vectors provides the tightest forward error bounds for the Ritz values. In view of the bound (3.2), it is natural to attempt to minimize $\sqrt{\left\|B^{-1}\right\|_{2}}\left\|r_{i}\right\|_{2}$ for $i=1,2, \ldots, k$. We do this by the following approach.

Suppose we have a set of computed Ritz vectors $\widehat{W} \in \mathbb{C}^{N \times k}$ such that $\widehat{W}^{H} A \widehat{W}=$ $\theta_{0} I_{k}$ and $\widehat{W}^{H} B \widehat{W}=I_{k}$. Recall that we can replace $\widehat{W}$ by $\widehat{W} Q$ for any unitary matrix $Q$. In our approach, we compute $\widehat{R}=A \widehat{W}-\theta_{0} B \widehat{W}$ and its SVD: $\widehat{R}=U_{R} \Sigma_{R} V_{R}^{H}$, where $\Sigma_{R}=\operatorname{diag}\left(\sigma_{1}(\widehat{R}), \ldots, \sigma_{k}(\widehat{R})\right)$ with $0 \leq \sigma_{1}(\widehat{R}) \leq \cdots \leq \sigma_{k}(\widehat{R})$. Then define $W$ and $R$ by $W=\widehat{W} V_{R}$ and $R=\widehat{R} V_{R}=U_{R} \Sigma_{R}\left(=A W-\theta_{0} B \bar{W}\right)$. This is equivalent to letting $Q=V_{R}$.

Note that this choice $Q=V_{R}$ is optimal in the sense that denoting $\bar{R}=\widehat{R} Q$, it minimizes $\|\bar{R}(:, 1: i)\|_{2}$ over all unitary $Q$ for all integers $i \leq k$. To see this, we use the property of singular values $[14$, p. 68] that for any matrix $X$, its $i$ th smallest singular value $\sigma_{i}(X)$ is characterized by

$$
\sigma_{i}(X)=\min _{\operatorname{dim}(S)=i} \max _{\substack{w \in S \\\|w\|_{2}=1}}\|X w\|_{2}
$$

Using (3.8) and denoting by $S_{i}$ the subspace spanned by the first $i$ columns of $I_{k}$, we have

$$
\|\bar{R}(:, 1: i)\|_{2}=\max _{\substack{w \in S_{i} \\\|w\|_{2}=1}}\|\bar{R} w\|_{2}=\max _{\substack{w \in S_{i} \\\|w\|_{2}=1}}\|\widehat{R} Q w\|_{2}=\max _{\substack{v \in Q S_{i} \\\|v\|_{2}=1}}\|\widehat{R} v\|_{2} \geq \sigma_{i}(\widehat{R})
$$

because $\operatorname{dim}\left(S_{i}\right)=\operatorname{dim}\left(Q S_{i}\right)=i$. Since $\|R(:, 1: i)\|_{2}=\sigma_{i}(\widehat{R})$, we see that the equality in (3.9) is attained for all integers $i \leq k$ when $Q=V_{R}$, so the claim is proved.

Now, consider $W_{2} \in \mathbb{C}^{N \times(N-k)}$ such that $W_{1}=\left[\begin{array}{ll}W & W_{2}\end{array}\right]$ satisfies $W_{1}^{H} B W_{1}=I_{N}$ (such $W_{1}$ exists, which can be obtained for example by $B$-orthonormalizing a nonsingular matrix $\left.\left[W \widehat{W}_{2}\right]\right)$. Then, we have

$$
W_{1}^{H} A W_{1}=\left[\begin{array}{cc}
\theta_{0} I & R_{2}^{H} \\
R_{2} & A_{22}
\end{array}\right],
$$

where $R_{2}=W_{2}^{H} R$. The matrix $W_{1}^{H} A W_{1}$ and the pencil $A-\lambda B$ have the same eigenvalues. Here, since we know that $\|R(:, 1: i)\|_{2}=\sigma_{i}(\widehat{R})$, we have

$$
\left\|R_{2}(:, 1: i)\right\|_{2} \leq\|R(:, 1: i)\|_{2}\left\|W_{2}\right\|_{2}=\sigma_{i}(\widehat{R})\left\|W_{2}\right\|_{2} \leq \sigma_{i}(\widehat{R}) \sqrt{\left\|B^{-1}\right\|_{2}},
$$

for $i=1, \ldots, k$. Here we used $\left\|W_{2}\right\|_{2} \leq\left\|W_{1}\right\|_{2}=\left\|B^{-1 / 2}\right\|_{2}$. Then, by using Weyl's theorem, we can conclude that for any integer $i \leq k$, there are at least $i$ eigenvalues of the pencil $A-\lambda B$ that satisfy

$$
\left|\lambda-\theta_{0}\right| \leq \sigma_{i}(\widehat{R}) \sqrt{\left\|B^{-1}\right\|_{2}} .
$$

Note that we only need to compute the singular values of $\widehat{R}$ (besides an estimate of $\left.\left\|B^{-1}\right\|\right)$ to get (3.11). Note also that for $i=1$, (3.11) is equivalent to (3.2) obtained 
by substituting the residual vector $R(:, 1)$, which is the smallest possible error bound for the Ritz value $\theta_{0}$ one can get using (3.2). Our bound (3.11) gives bounds also for $i \geq 2$.

\subsection{Simple example}

Let us return to the pencil (3.4), and again consider the case $C_{1}=0.1\left[\begin{array}{ll}1 & 1 \\ 1 & 1\end{array}\right]$ and $C_{2}=0.1\left[\begin{array}{ll}1 & 0 \\ 0 & 1\end{array}\right]$ to demonstrate the approach described above. Suppose the Ritz vectors $\widehat{W}=\left[\widehat{w}_{1}, \widehat{w}_{2}\right]$ (not unique: for example, $\hat{W}$ can be $\left[w_{1}, w_{2}\right]$ or $\left[\widetilde{w}_{1}, \widetilde{w}_{2}\right]$ in Sect. 3.2) are computed so that $\widehat{W}^{H} A \widehat{W}=\theta_{0} I_{2}\left(=I_{2}\right)$ and $\widehat{W}^{H} B \widehat{W}=I_{2}$. Our approach computes $\Sigma_{R}$ in the $\operatorname{SVD} \widehat{R}=(A-B) \widehat{W}=U_{R} \Sigma_{R} V_{R}^{H}$. In this case, $\Sigma_{R}=\operatorname{diag}\left(\sigma_{1}(\widehat{R}), \sigma_{2}(\widehat{R})\right)=\operatorname{diag}\left(10^{-3}, 10^{-1}\right)$, regardless of the choice of $\widehat{W}$. Then, again using $\left\|B^{-1}\right\|_{2} \leq 1 / 0.9$, we use (3.11) to conclude that one eigenvalue satisfies $|\lambda-1| \leq \sqrt{\left\|B^{-1}\right\|_{2}} \sigma_{1}(\widehat{R})<1.06 \times 10^{-3}\left(=\delta_{1}\right)$, and that two eigenvalues satisfy $|\lambda-1| \leq \sqrt{\left\|B^{-1}\right\|_{2}} \sigma_{2}(\widehat{R})<1.06 \times 10^{-1}\left(=\delta_{2}\right)$.

The true eigenvalues of $A-\lambda B$ are $\simeq\left(1-10^{-6}, 1-10^{-2}, 2,2+2 \times 10^{-2}\right)$. Note that the sensitivity difference between the two eigenvalues close to 1 is a result of the difference between $\sigma_{1}(\widehat{R})$ and $\sigma_{2}(\widehat{R})$, which is justified by observing that if we replace the $(1,1)$ elements of $A$ and $B$ by 1 , then $\sigma_{1}(\widehat{R})=\sigma_{2}(\widehat{R})=0.1$, and the eigenvalues become $\simeq\left(1-9.7 \times 10^{-3}, 1-10^{-2}, 2,2+4 \times 10^{-2}\right)$, so both eigenvalues exhibit similar sensitivities.

Unfortunately our error estimates $\delta_{1}$ and $\delta_{2}$ for the two eigenvalues close to 1 are both overestimates, and in particular we observe that their squares $\delta_{1}^{2}, \delta_{2}^{2}$ seem to be accurate estimates of the true errors. This behavior is rather general, and in fact was true for all randomly constructed $C_{1}$ and $C_{2}$ that we tried. However we cannot make this observation precise; the known quadratic error bound $\left\|r_{i}\right\|_{2}^{2} /$ gap in [1] is not helpful here, because $g a p \simeq 10^{-2}$ is small (or unavailable in practice when a multiple Ritz value exists) and the bounds will not be improved. We can also apply quadratic residual bounds [8,9] to the matrix (3.10), in which case we get an error bound $\left\|R_{2}\right\|^{2} / 1=10^{-2}$. However this is an error bound for both eigenvalues, and does not describe the less sensitive eigenvalue. A rigorous explanation for the observation is an open problem.

Acknowledgements I am grateful to the referees for their helpful suggestions, which greatly improved the clarity.

Open Access This article is distributed under the terms of the Creative Commons Attribution Noncommercial License which permits any noncommercial use, distribution, and reproduction in any medium, provided the original author(s) and source are credited.

\section{References}

1. Bai, Z., Demmel, J., Dongarra, J., Ruhe, A., van der Vorst, H.: Templates for the Solution of Algebraic Eigenvalue Problems: A Practical Guide. SIAM, Philadelphia (2000)

2. Barlow, J., Slapnicar, I.: Optimal perturbation bounds for the Hermitian eigenvalue problem. Linear Algebra Appl. 309(1-3), 19-43 (2000) 
3. Demmel, J.: Applied Numerical Linear Algebra. SIAM, Philadelphia (1997)

4. Golub, G.H., Van Loan, C.F.: Matrix Computations. The Johns Hopkins University Press, Baltimore (1996)

5. Higham, N.J.: Functions of Matrices: Theory and Computation. SIAM, Philadelphia (2008)

6. Knyazev, A.V.: Toward the optimal preconditioned eigensolver: locally optimal block preconditioned conjugate gradient method. SIAM J. Sci. Comput. 23(2), 517-541 (2001)

7. Kressner, D., Pelaez, J.M., Moro, J.: Structured Holder condition numbers for multiple eigenvalues. SIAM J. Matrix Anal. Appl. 31(1), 175-201 (2009)

8. Li, C.K., Li, R.C.: A note on eigenvalues of perturbed Hermitian matrices. Linear Algebra Appl. 395, 183-190 (2005)

9. Mathias, R.: Quadratic residual bounds for the Hermitian eigenvalue problem. SIAM J. Matrix Anal. Appl. 19(2), 541-550 (1998)

10. Nakatsukasa, Y.: Absolute and relative Weyl theorems for generalized eigenvalue problems. Linear Algebra Appl. 432, 242-248 (2010)

11. Parlett, B.N.: The Symmetric Eigenvalue Problem. SIAM, Philadelphia (1998)

12. Shao, M.: Perturbation analysis for symmetric-definite pencils. Private communication (2009)

13. Sleijpen, G.L.G., Van der Vorst, H.A.: A Jacobi-Davidson iteration method for linear eigenvalue problems. SIAM Rev. 42(2), 267-293 (2000)

14. Stewart, G.W.: Matrix Algorithms, vol. I, Basic Decompositions. SIAM, Philadelphia (1998)

15. Stewart, G.W., Sun, J.G.: Matrix Perturbation Theory. Academic Press, San Diego (1990)

16. Stewart, G.W., Zhang, G.: Eigenvalues of graded matrices and the condition numbers of a multiple eigenvalue. Numer. Math. 58(7), 703-712 (1991)

17. Sun, J.G.: On worst-case condition numbers of a nondefective multiple eigenvalue. Numer. Math. 69(3), 373-382 (1995) 